\title{
Une brique inscrite d'Adad-apla-iddina provenant de Larsa
}

\author{
Régis Vallet et Camille Lecompte ${ }^{1}$
}

On sait que depuis plus de vingt ans maintenant le marché est inondé d'objets provenant des grands sites d'Irak, mais parfois, l'un de ceux qui vient à faire surface a été fortuitement trouvé par un simple visiteur, bien avant la période des grands pillages récents. ${ }^{2}$ C'est le cas de la brique inscrite que nous publions ici, ramassée à la surface de Larsa vers la fin des années 70 et actuellement conservée dans une collection privée ${ }^{3}$. Les informations transmises et les caractéristiques de l'objet ne laissent aucun doute sur son authenticité et sa provenance. Dans la mesure où l'on ne possédait jusqu'à présent aucune photographie de son inscription, bien connue par ailleurs, il nous a paru utile de rendre accessible cet exemplaire supplémentaire (Fig. 1), accompagné d'une photographie inédite de celui trouvé par A. Parrot en 1967 et publié par M. Birot (Fig. 3) ${ }^{4}$.

\section{L'objet}

L'état de conservation de la brique est relativement bon, seule sa face supérieure, inscrite, présentant des desquamations superficielles (notamment au niveau des $4^{\mathrm{e}}$ et $7^{\mathrm{e}}$ lignes de l'inscription) et une érosion certainement due à une exposition prolongée aux conditions atmosphériques, éoliennes en particulier, conjuguées peut-être à l'action de sels solubles contenus dans le matériau (auréoles jaunâtres). La pâte, très bien cuite (vers $800^{\circ}$ ), en atmosphère oxydante, est absolument typique des matériaux argileux de texture fine et de couleur beige verdâtre que l'on trouve dans la région de Larsa, et contient une forte quantité de dégraissant, à la fois végétal et minéral (grains siliceux brun rouge à rouge). La brique, moulée, de format carré, est d'un module similaire à celui des exemplaires déjà connus : 28,5 cm de côté pour $6 \mathrm{~cm}$ d'épaisseur en moyenne, avec un cartouche de 14,5 x $9 \mathrm{~cm}(8,5 \mathrm{~cm}$ seulement à son extrémité inférieure $)^{5}$. Elle présente toutefois d'importantes déformations structurelles, dues à un manque de matériau et une exécution très peu soignée : sa face sud (selon l'orientation de l'inscription) ne mesure que 26,5 cm de long (contre $29 \mathrm{~cm}$ à l'O.) et l'épaisseur de son angle $\mathrm{SE}$ tombe à 4,5 $\mathrm{cm}$ (contre $6,5 \mathrm{~cm}$ au NE). Manque de pâte que l'on a cherché à compenser

\footnotetext{
${ }^{1}$ C.N.R.S., UMR 7041 ArScAn-VEPMO, M.A.E., Nanterre. Nous remercions nos collègues du service des archives de la Maison Archéologie et Ethnologie, Elisabeth Bellon, Aurélie Montagne-Bôrras et François Rimelen, pour l'aide qu'ils nous ont apportée, ainsi que Christine Pariselle, pour son expertise du matériau. Nous sommes également redevables à D. Shibata de son aide précieuse à l'interprétation du terme šu - $\mathrm{il}_{2}$. C'est avec plaisir que nous nous associons à l'hommage rendu à notre ami Olivier Rouault, que nous avons rencontré en Irak il y a quelques années (c'était avant la guerre du Koweit), puis sur nos sites respectifs de Syrie et à nouveau d'Irak plus récemment. Olivier Rouault, parfait gentleman par ailleurs, a formé plusieurs générations de chercheurs à 1'Université de Lyon II, associant toujours étroitement histoire et archéologie, disciplines qu'il pratique conjointement avec le même bonheur. Qu'il accueille cette modeste note en gage de notre estime et de notre amitié.

${ }^{2}$ Pour un bref rappel de la situation des patrimoines syrien et irakien, Cf. Vallet et Varoutsikos 2016.

${ }^{3}$ De la même manière, une figurine ophidienne trouvée à la surface de Tell el 'Oueili nous a récemment été présentée pour expertise par son inventeur (Vallet et Pariselle 2015).

${ }^{4}$ Birot 1968, p. 246-247 ; photographie conservée au service des Archives de la M.A.E. de Nanterre et, provenant des carnets d'A. Parrot, intégrée aux archives de la Mission de Mari, avec la courtoisie de P. Butterlin. Les archives de la Mission de Larsa, également conservées à la MAE, ne contiennent pas de photographie des briques inscrites découvertes par J.-L. Huot, que nous remercions pour nous avoir autorisés à publier cet objet, découvert tout à fait indépendamment de la mission de Larsa mais à l'époque où il dirigeait celle-ci.

${ }^{5}$ Cf. Huot 1989, p. $32: 29$ × 29 × 5 cm, Birot 1968, p. 246 (sous la copie) :13,5 x 8 cm pour le cartouche. Voir également les tables remarquables de précision et particulièrement complètes établies par Sauvage 1998, p. 296-297 ( ${ }^{\circ}$. 1888-1902 pour l'ensemble des briques de l'époque d'Adad-apla-iddina, et 1888-1891 pour les briques du type présenté ici); cf. Sauvage 1998, p. 376 (nº 513) à propos de la « mise en œuvre » des briques de ce souverain.
} 
par l'inclusion d'un matériau de remplissage de substitution (gros nodule minéral gris). C'est clairement une fin de série, spécialement irrégulière, y compris en épaisseur. Dans la mesure où l'on sait qu'elle était destinée au carrelage de l'e.babbar (voir ci-dessous), il est possible que, bien qu'inscrite, mais impropre à l'usage destiné, elle ait été d'emblée écartée de ses semblables et recyclée dans d'autres constructions. Ce qui pourrait éventuellement expliquer, plutôt que du véritable remploi ou du pillage, qu'elle ait été retrouvée loin du temple. Quoi qu'il en soit, c'est par son inscription que l'objet présente un quelconque intérêt.

\section{L'inscription}

Cette inscription (B.2.8.9.), provenant bien entendu exclusivement de Larsa, a déjà fait l'objet de plusieurs publications, notamment celle de G. Frame (1995, p. 59-60), qui comporte les références bibliographiques à son sujet. Les exemplaires de cette inscription, tous découverts lors des fouilles menées sur le site, furent retrouvés soit en surface, soit in situ sur le carrelage de la cour I et du couloir III de la pièce $8^{6}$. Leur nombre exact reste indéfini dans tous les cas, seuls ceux découverts lors de la campagne de 1970 portant un numéro d'inventaire (L. 70.84), d'ailleurs générique. L'exemplaire publié ici (Fig. 1 et 2 ) est identique à ceux déjà connus :

\begin{tabular}{|c|c|c|}
\hline 1. & ${ }^{\mathrm{d}}$ Adad-'apla ${ }^{-}$ & Adad-apla-iddina \\
\hline 2. & $i$-din-nam & \\
\hline 3. & lugal $\mathrm{KA}_{2}$. DINGIR. ${ }^{\circ} \mathrm{RA}^{\top}$ & roi de Babylone \\
\hline 4. & ki -ma & \\
\hline 5. & saĝ-'gal’ & le $«$ premier $»$ \\
\hline 6. & ${ }^{\mathrm{d}} \mathrm{Utu}-\mathrm{ke}_{4}$ & de Šamaš \\
\hline 7. & riz-zi` $e_{2}-a$ & le mur du temple \\
\hline 8. & $e_{2}$ babbar-ra & de l'e-babbar \\
\hline 9. & dur ${ }_{2}$ šu- $^{-} \mathrm{il}_{2}{ }^{\top}$ & lieu élevant la main (?) \\
\hline 10. & ba- ${ }^{r n i-i n}{ }^{\urcorner}-A K$ & il a fait faire \\
\hline
\end{tabular}

\section{Commentaires}

D. Arnaud puis G. Frame ayant déjà relevé les aspects philologiques les plus importants, l'on se contentera ici de commenter les quelques termes de cette inscription retenant notre attention.

\footnotetext{
${ }^{6}$ Pour plus de détails, voir Huot 2014, p. 132 et, à propos de la pièce 8, p. 57. Les exemplaires ramassés en surface sont mentionnés dans Birot 1968, p. 242 (campagne de 1967, un exemplaire, apparemment sans numéro d'inventaire) ; Arnaud 1976, p. 48 (campagne de 1974, exemplaires en nombre indéterminé, sans numéro d'inventaire). Ceux in situ sont répertoriés dans : Arnaud 1971, p. 291 (campagne de 1970, " dallage de la cour intérieure du temple », nombre indéterminé, tous regroupés sous le numéro d'inventaire L. 70.84, comme rappelé cidessous) ; Calvet, Charpin, Cleuziou, Forest et Huot 1976, p. 17 (un exemplaire retrouvé en 1974 sur le couloir « nord-est», nIII, de la pièce 8) ; Arnaud 1976, p. 49 (en dépit de l'absence de précision de l'auteur, la brique $\mathrm{n}^{0} 23$ - dont le nombre d'exemplaires n'est pas mentionné - doit correspondre à celle retrouvée dans le couloir de la pièce 8); Arnaud 1981, p. 42 (résumé des découvertes). Voir également Sauvage 1998 , p. $296-297$ et 376.
} 
3. saĝ-gal : notre exemplaire confirme bien l'interprétation de D. Arnaud de saĝ-gal (contra Frame, RIMB p. 60 note 1). L'équivalence avec $a s ̌ a r e ̄ d u$, établie sûrement par diverses sources (cf. CAD A/2, p. 416-417), ne semble pas attestée dans les titulatures des souverains de la même dynastie.

7-8. iz-zi : ce terme bien connu est équivalent à l'akkadien igārum, «mur, paroi », cf. Proto-Kagal Bil Section E 50. Le type d'ouvrage auquel il se rapporte dans le contexte de l'inscription reste incertain : alors que G. Frame suggère qu'il s'agit d'une enceinte, enclosure, sans préciser si celle-ci correspond à la limite du complexe de l'e-babbar ou aux murs de la cour I, le terme nous semble davantage tendre vers cette dernière hypothèse. À titre de comparaison, les travaux menés par J.-L. Huot ne semblent avoir mis en évidence que la pose par Adad-apla-iddina du carrelage de la cour I (ainsi que dans le couloir III de la pièce 8), le bâtiment ayant dans l'ensemble fait l'objet d'une restauration sous les rois kassites $^{7}$. J.-L. Huot observa en outre que la pièce 14 associée à la même cour connut, lors d'une troisième phase de son histoire, une surélévation de sa partie centrale, qu'il serait possible d'attribuer à Adad-apla-iddina, conformément aux travaux menés dans le reste du secteur ${ }^{8}$. Il est par conséquent légitime de se demander si l'inscription ne tend pas à exagérer quelque peu l'ampleur des travaux entrepris par ce dernier souverain.

Dans la mesure où le terme $e_{2}$ de la ligne 7 est déjà noté avec un cas (-a), on ne sait trop comment construire un génitif tenant sur une seule chaîne nominale avec le nom de l'e $e_{2}$-babbar, qui semble d'ailleurs écrit, dans les inscriptions de la II $^{\mathrm{e}}$ Dynastie d'Isin, de manière figée $\mathrm{e}_{2}$-babbar-ra, quel que soit sa fonction grammaticale. On pourrait donc à la rigueur comprendre la ligne 8 comme « dans l'ebabbar », avec un locatif.

9. Bien que D. Arnaud, suivi par G. Frame, ait identifié le terme šu - $\mathrm{il}_{2}$ avec une "prière », renvoyant au genre bien connu des prières et rituels dits šu-il $1_{2}-\mathrm{la}_{(2)}$, il est probable que cette inscription se réfère plus simplement au geste proprement dit consistant à lever la main, ainsi d'ailleurs que cela ressortait de la traduction plus prudente de Birot, «temple de l'élévation de sa main ». Comme nous le suggère D. Shibata (communication personnelle), l'emploi de šu - $\mathrm{il}_{2}$ et non de la forme šu-il $\mathrm{l}_{2}-\mathrm{la}_{(2)}$ semble démontrer qu'il conviendrait de traduire le terme dur 2 šu-il 2 comme «lieu élevant la main ${ }^{9}-$ ou « lieu dont la main est levée » -, de manière similaire à l'étymologie du temple de Babylone $\mathrm{e}_{2}$-saĝ-il ${ }_{2}$, sans que cette expression n'entretienne de relation avec le type de prière et de rituel assurés par l'āšipum ou le kalû. On ne sait toutefois s'il s'agit d'une référence au geste employé lors des

\footnotetext{
${ }^{7}$ Huot 2014, p. 132. Il convient de noter que J.-L. Huot ne prit n'a pris en compte que la traduction offerte par Birot, qui omettait le terme iz-zi e $e_{2}$-a, pourtant clairement identifié plus tard par D. Arnaud comme se rapportant à un mur de l'e-babbar. Cette traduction, suivie par G. Frame ainsi que dans le présent article, conduit par conséquent à réévaluer l'ampleur des travaux opérés par ce roi, bien qu'il soit tout à fait envisageable que l'inscription ne concorde pas avec la réalité et qu'Adad-apla-iddina se soit contenté de restaurations plus légères.

${ }^{8}$ A ce propos, voir Huot 2014, p. 73 et 136 : « On peut lui [à Adad-apla-iddina] attribuer également la surélévation (état ancien) de la partie centrale de la pièce 14, qui correspond à la surélévation du niveau de la cour I consécutive à la pose de ce carrelage ». L'auteur n'exclut pas en outre que la troisième phase des coffres à crapaudine, consistant en un appareil de «briques minces », dans la pièce 14, soit postérieure à Burnaburiaš, cf. Huot 2014, p. 80.

${ }^{9}$ À propos de l'étymologie du terme šu - il 2 , cf. Shibata à paraître ; Frechette 2012, p. 11-16.
} 
audiences ou à une autre réalité ${ }^{10}$. En dépit des divergences de translittérations du signe considéré comme TUŠ par D. Arnaud et $\mathrm{DUR}_{2}$ par G. Frame, nous avons opté, en raison de la forme graphique observable sur tous les exemplaires connus, pour la seconde possibilité, même si TUŠ s'avèrerait justifié.

10. Noter l'emploi des préfixes ba-ni pour le causatif.

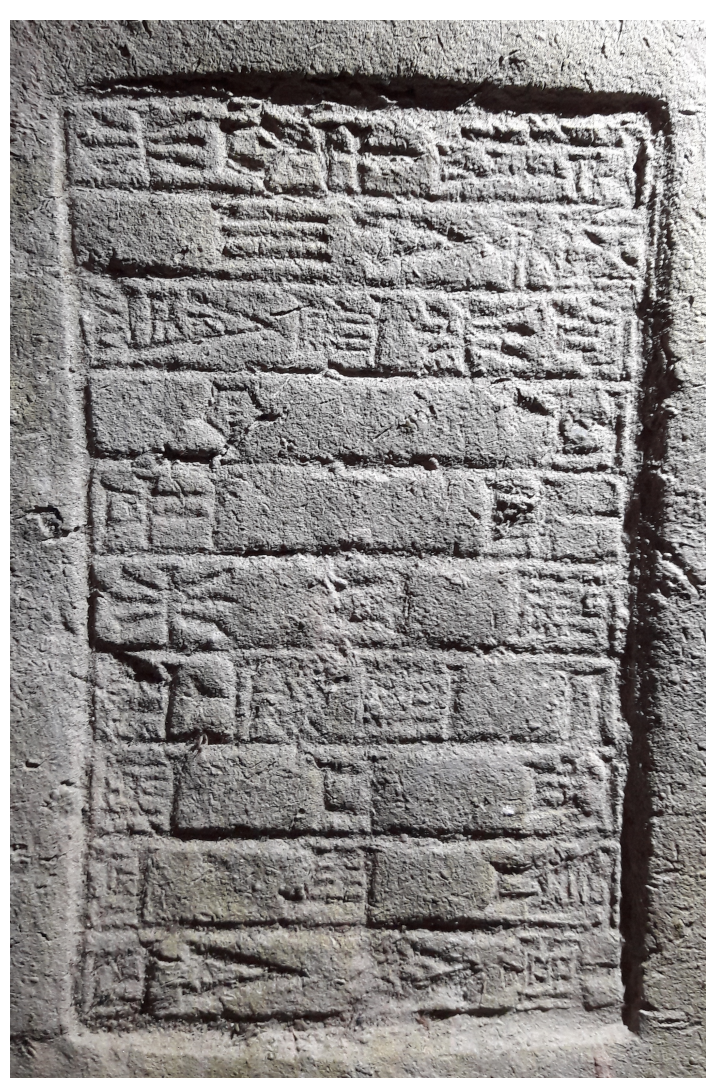

Fig. 1 : Photographie de l'inscription

(Cliché R. Vallet, Mission de Larsa-Oueili)

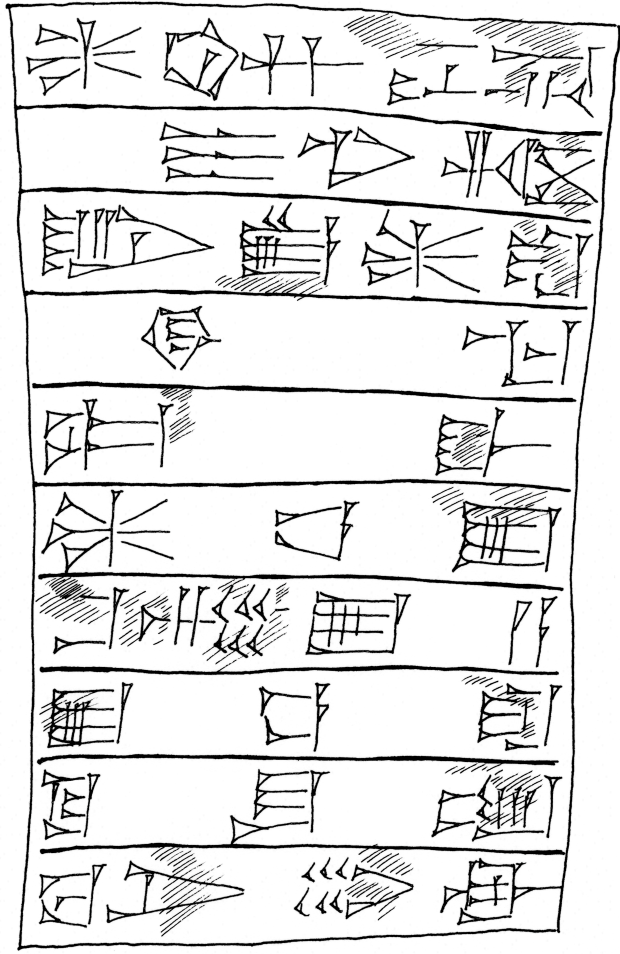

Fig. 2 : Copie de l'inscription (C. Lecompte)

\footnotetext{
${ }^{10} \mathrm{Si}$ toutefois l'inscription d'Adad-apla-iddina se rapportait au lieu lié au rituel ou à la prière šu'ila, elle constituerait une information intéressante sur les modalités de sa réalisation. D'après les études de Frechette 2012, p.149-152, relatives aux prières šu'ila akkadiennes, celles-ci étaient accomplies dans des lieux purifiés, sur le toit de bâtiments, dans des structures provisoires ou dans des lieux isolés pour des raisons de pureté.
} 


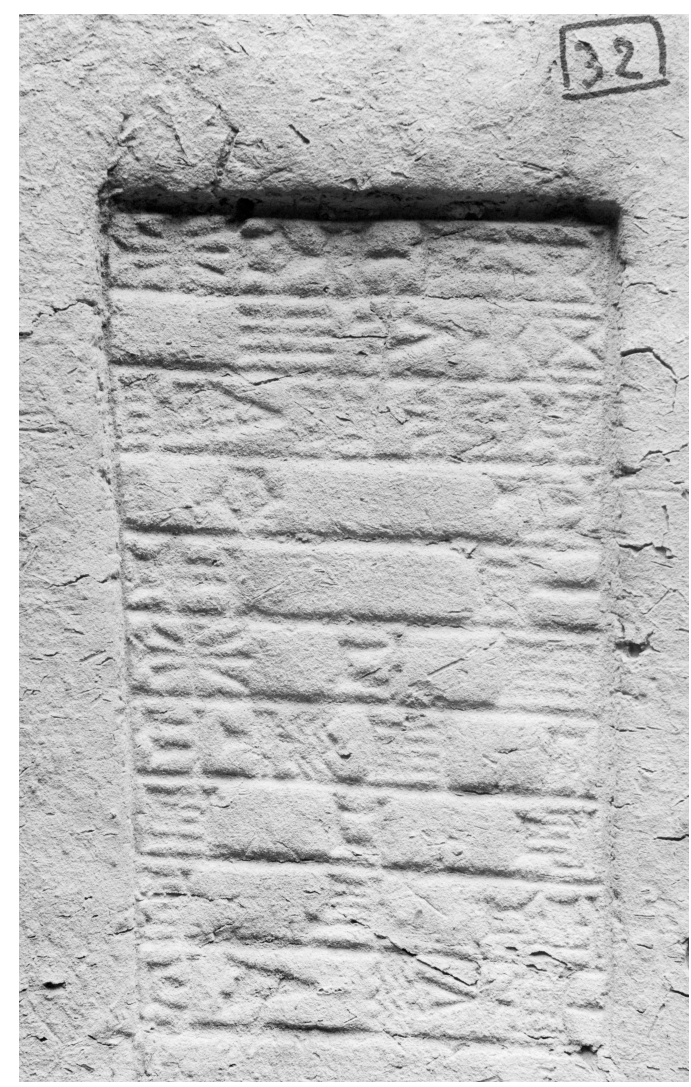

Fig. 3 : Photographie de l'exemplaire publié par M. Birot en 1968 ( $n^{\circ} 13458$, carnet IV des photographies d'A. Parrot, service des archives de la MAE)

\section{Bibliographie}

ARNAUD, D. 1971, «Catalogue des textes trouvés au cours des fouilles et explorations régulières de la maison à Tell Senkereh-Larsa en 1969 et $1970 »$, Syria. 48, p. 289-293

ARNAUD, D. 1976, «Catalogue des textes et des objets inscrits trouvés au cours de la sixième campagne », Syria 53, p. 47-81

ARNAUD, D. 1981, « Annexe : Textes divers concernant le royaume de Larsa », Syria 58, p. 70-100

BIROT, M. 1968, « Découvertes épigraphiques à Larsa (campagnes 1967)», Syria 45, p. 241-247

Calvet, Y., Charpin, D., Cleuziou, S., Forest J.-D. et Huot, J.-L. 1976, « Rapport préliminaire sur la sixième campagne de fouilles », Syria 53, p. 1-45

FrAme, G. 1995, Rulers of Babylonia : From the Second Dynasty of Isin to the End of Assyrian Domination (1157-612 BC), RIMB 2

FreChetTE, C. 2012, Mesopotamian Ritual-prayers of "Hand-lifting" (Akkadian Suillas). An Investigation of Function in Light of the Idiomatic Meaning of the Rubric, AOAT 379

HuOT, J.-L. 1989, Larsa. Travaux de 1985, ERC

HuOT, J.-L. 2014, L'E.babbar de Larsa aux II et $I^{e r}$ millénaires, BAH 205

SaUvage, M. 1998, La brique et sa mise en ceuvre en Mésopotamie. Des origines à l'époque achéménide, ERC

SHIBATA, D. À paraître, Die Šu ila Gebete im Emesal, Heidelberger Emesal-Studien, Harrassowitz 
VAllet R. et Pariselle C. 2015 « Une figurine ophidienne inédite de Tell el 'Oueili », Matoian V. (éd.) Mélanges offerts à Yves Calvet, RSO XXII, p. 343-352

VAllet, R. et VAROUTSiKos B. 2016, « Palmyre, symbole de la destruction du patrimoine culturel de Proche-Orient », Al-Maqdissi M. et Ishaq E. (éds), La Syrie et le désastre archéologique du ProcheOrient, Beyrouth, p. 115-118 [N. B. : par suite d'une erreur de l'éditeur, les illustrations publiées ne correspondent pas à celles de l'article] 